\title{
THE TREATMENT WITH WATER SEAL CHEST DRAIN (PDU) IN TYPE 2 DIABETES MELLITUS PATIENT WITH RIGHT HYDROPNEUMOTHORAX OF TUBERCULAR AETIOLOGY WITH PERSISTENT RIGHT PNEUMOTHORAX WITH BPF AFTER FAILURE OF DECORTICATION
}

\author{
D. Sudeena ${ }^{1}$, S. B. Lal'2, Sree Karuna Murthy Kolli ${ }^{3}$
}

1Associate Professor, Department of Pulmonary Medicine, Osmania Medical College, Hyderabad, Andhra Pradesh, India. ${ }^{2}$ Registrar, Dr. NTR University of Health Sciences, Vijayawada \& Former Professor \& HOD Department of Pulmonary Medicine, Siddhartha Medical College, Vijayawada, Andhra Pradesh, India.

${ }^{3}$ Associate Professor, Department of Community Medicine, Dr. PSIMS \& RF Krishna District.

\begin{abstract}
A male patient, D. Sanjeev Rao, aged 50 years, lorry driver by occupation, residing at Vijayawada, AP, presented with Rt. sided chest pain, cough, severe breathlessness, low-grade fever since one day. He was admitted in the casualty under emergency condition and was resuscitated with oxygen inhalation. On inspection, trachea was deviated to right side. Chest was bilaterally asymmetrical. Intercostal tube with underwater seal drainage on right side was found. Right side of chest shows loss of lung volume with wasting of muscles, drooping of shoulder, narrowing of intercostal spaces with crowding of ribs. Apical impulse appears to be shifted to right side. On palpation respiratory movements diminished all over the right chest with increased vocal fremitus over the middle of chest and decreased in base. On percussion dull note was present in base, resonant in middle of the chest. On auscultation, amphoric breathing with metallic quality were heard on right side. CVS-S1, S2 present. On percussion, heart borders were in normal limits. ICD tube column moving continuously. CX-Ray PA view with right pneumothorax encysted with thickened pleural margins with right upper lobe collapse. Patient underwent right decortication on $30^{\text {th }}$ June 2015. Since then lung was not expanded, but landed into continuous air leakage with bronchopleural fistula with persistent right pneumothorax pocket. Patient was diagnosed as hydropneumothorax. ${ }^{1}$ on 1-6-15, treated with intercostal tube. ${ }^{2}$ drainage, but was failed with occurrence of bronchopleural fistula. ${ }^{3}$

Later, he underwent decortication. ${ }^{1}$ and presented with persistent pneumothorax. ${ }^{4}$ Patient is a known case of type- 2 diabetic, on medication with Human Mixtard insulin for the past 5years. Diabetes was under control. Pus from ICD bag sent for culture for AFBnot detected by culture, confirmed by "MycoReal" real time PCR by SRL diagnostic test.
\end{abstract}

\section{KEYWORDS}

BPSK Modulator, Convolutional Encoder, CORDIC, DS-CDMA/CI, FPGA.

HOW TO CITE THIS ARTICLE: Sudeena D, Lal SB, Kolli SKM. The treatment with water seal chest drain (PDU) in type 2 diabetes mellitus patient with right hydropneumothorax of tubercular aetiology with persistent right pneumothorax with BPF after failure of decortication. J. Evolution Med. Dent. Sci. 2016;5(7):333-335, DOI: 10.14260/jemds/2016/72

\section{INTRODUCTION}

A 50-year-male patient with persistent air leakage on bronchopleural fistula on right side of chest since 3 months suffering with right chest pain, cough, sob with decreased air entry on right side with decreased respiratory movements, right mediastinal shift. Type 2 diabetes mellitus under control with insulin. Patient underwent decortication for right lung BPF, but patient recur with encysted pneumothorax. After 3 months, patient diagnosed and treated with PDU under the guidance of CT surgeon. Sputum for AFB-negative on 14-082015, KOH wet mount negative for fungal elements.

\section{DISCUSSION}

Hydropneumothorax (Definition) concurrent presence of air and fluid in the pleural space.

Occurrence of tuberculosis. 5 in case of diabetes mellitus and increased susceptibility of patients with diabetes mellitus to develop tuberculosis is due to:

- Neutrophilic dysfunction.

Financial or Other, Competing Interest: None.

Submission 03-10-2015, Peer Review 14-10-2015,

Acceptance 20-10-2015, Published 23-01-2016.

Corresponding Author:

Dr. D. Sudeena,

Associate Professor

Department of Pulmonary Medicine,

Osmania Medical College, Hyderabad,

Andhra Pradesh, India.

E-mail: drsudeenaravi@gmail.com

DOI: $10.14260 /$ jemds $/ 2016 / 72$
- Impaired cytokine production (INF-gamma/IL-14/TNFalpha).

- Thickened alveolar epithelium and pulmonary basal lamina.

- Reduced pulmonary diffusion capacity.

- Decreased lung volumes and elastic recoil diabetes mellitus accounted $14.8 \%$ of pulmonary $\mathrm{TB}, 20.2 \%$ sputum positive TB.

\section{DECORTICATION}

Surgical removal of the surface layer, membrane or fibrous cover of an organ. The procedure is usually performed when lung is covered by a thick elastic pleural peel restricting lung expansion.

\section{Indications}

1. Pleural peel present for $>6$ weeks.

2. Lung symptoms are disabling.

3. Radiological evidence of trapped lung.

\section{Contraindications}

1. Presence of pleural space infection.

2. Large airway stenosis.

3. Severe chest wall infection.

4. Terminal disease.

5. Coagulopathy.

Increased post-operative decortication risk

1. Advanced age.

2. Coronary artery disease. 
3. COPD.

4. Asthma.

5. Extensive lung resection.

6. Emergency procedure.

7. Morbid obesity.

8. Prolonged surgery.

9. Extensive bleeding during surgery.

10. Smoking.

\section{If any of them is present following must be ordered}

- Thorough history, PFT, Spirometry, ABG, Cardiopulmonary exercise test.

- Early postoperative complications leads to re-operation.

- Prolonged air leakage, Bleeding, Empyema, Co-existing diabetes or immune-compromised states, Lean body mass index, Smoking history, Younger age group, Secondary pneumothorax.

- Type of surgery is decided by the surgeon.

\section{NOTE}

Pulmonary wedge resection seems to protect against recurrence while pleurodesis and/or pleurectomy in addition to wedge resection do not. Depending upon the clinical indication, once a chest tube is placed a PDU. 6 may be attached to provide suction and/or water seal to prevent back flow of air into the pleural space. The same resistance considerations in choosing a chest tube need to be assessed for the connecting tubing and the multi-chambered drainage device. ${ }^{7}$ comprising a PDU. Commercially available in their flow rates and the accuracy of delivered negative pressures. An assessment of commercial PDUs available in the United States notes air flow rate capabilities at $-20 \mathrm{com}$ of water pressure vary with ranging with 10.8 to $42.1 \mathrm{~L} / \mathrm{min}$. The accuracy of the measured level of suction delivered varies significantly, but may be of a magnitude that is not clinically significant.

The assessed commercially available PDUs are all based upon the traditional three chamber device. This three chamber device is now commercially packaged into compartmentalized, durable, convenient single units providing easy mobility and specimen collection. The three compartments sequentially include the collection compartment (Frequently with sampling ports) to trap liquid material and suspended debris from the patients pleural space while allowing any pleural air to pass through the next two compartments. The water seal compartment to prevent air flow back to the patient's pleural space and to allow detection of an air leak (BPF) and the manometer compartment to regulate the amount of negative pressure transmitted back to the patient from the valve suction device. Commercial PDUs are available as wet devices like Atrium Ocean water seal chest drain depending upon whether they have traditional water based manometer compartment or system based upon a spring loaded valve system.

Disposable Water Seal Chest Drain (Pleural Drainage Unit) with wet suction control and calibrated water seal.
Sterile fluid is required for water seal air leak detection. For suction control operation, sterile saline is required. The chest drain is non-pyrogenic and is for single patient use only. Models equipped with a patient tube in line connector provide convenient system change out.

\section{Indications}

1. Evacuate air and fluid from the chest or the mediastinum.

2. Help re-establish lung expansion and restore breathing dynamics.

3. Facilitate post-operative collection and reinfusion of autologous blood from the patients' pleural cavity or mediastinal area.

\section{CONCLUSION}

Although awareness regarding basic aspects of TB was adequate, knowledge about prevention, curability and free treatment of TB should be stressed. Periodic KAP (Knowledge, Attitude and Practice) surveys will help in identifying the deficiencies in knowledge and practices of the community, thereby providing clues to improve performance of RNTCP. In uncontrolled T2 DM, there is more risk of occurrence of TB, so education and careful followup of the patient and diet control along with insulin or suitable OHA (Oral Hypoglycemic Agents) is mandatory. To avoid failure of decortication in such cases, Surgeon must be well aware of indications of surgery and also adequate control of T2 DM.

In order to avoid recurrent surgical management in this case of persistent pneumothorax with BPF, the Pulmonologist is expected to be aware of alternative management with Disposable Water Seal devices like PDU. It helps to avoid further collapse of lung and also to allow lung expansion. Patient presented with Hydropneumothorax. The Author hereby Thank the Dept. of CT Surgery, HOD and Professor Dr. Sai Kumar, Dept. of Pulmonary Medicine, OMC, Hyderabad and Post Graduate students Dr. G. Vedaranya of Gandhi Medical College, Dr. Venugopal and Dr. Keerthi of Osmania Medical College, Dept. of Pulmonary Medicine.

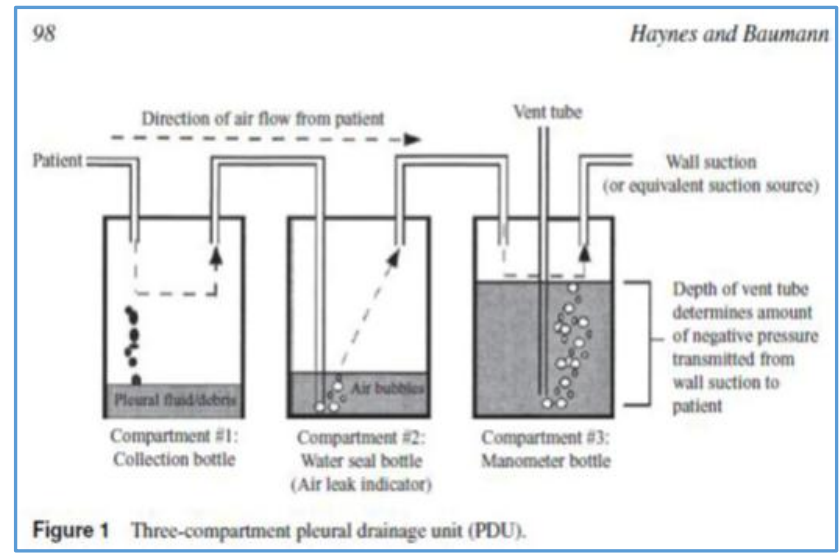




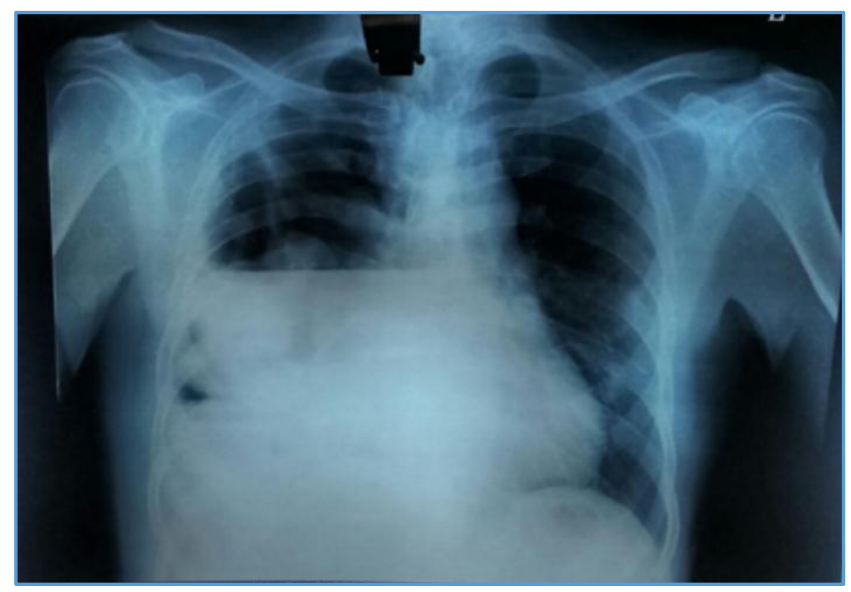

Patient presented with Hydropneumothorax

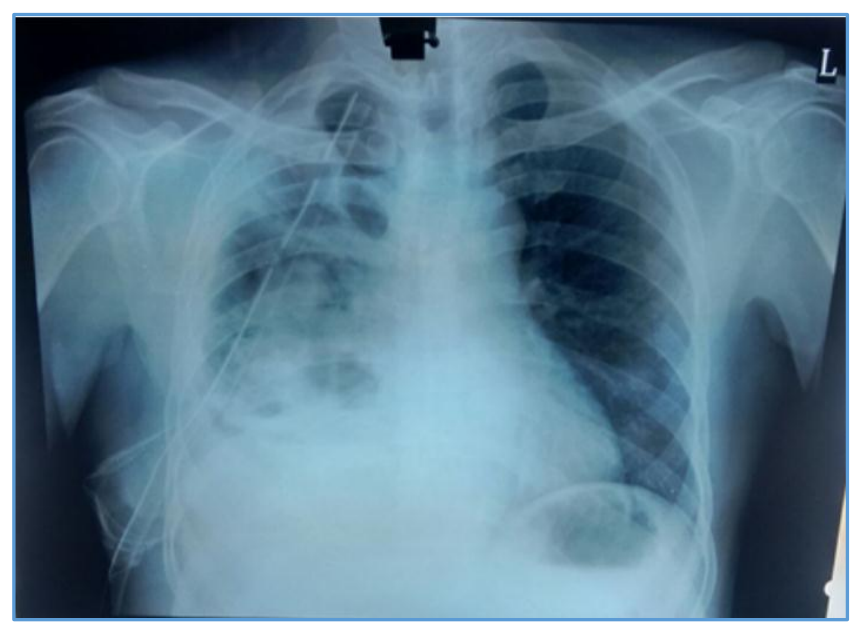

ICD tube in situ with BPF

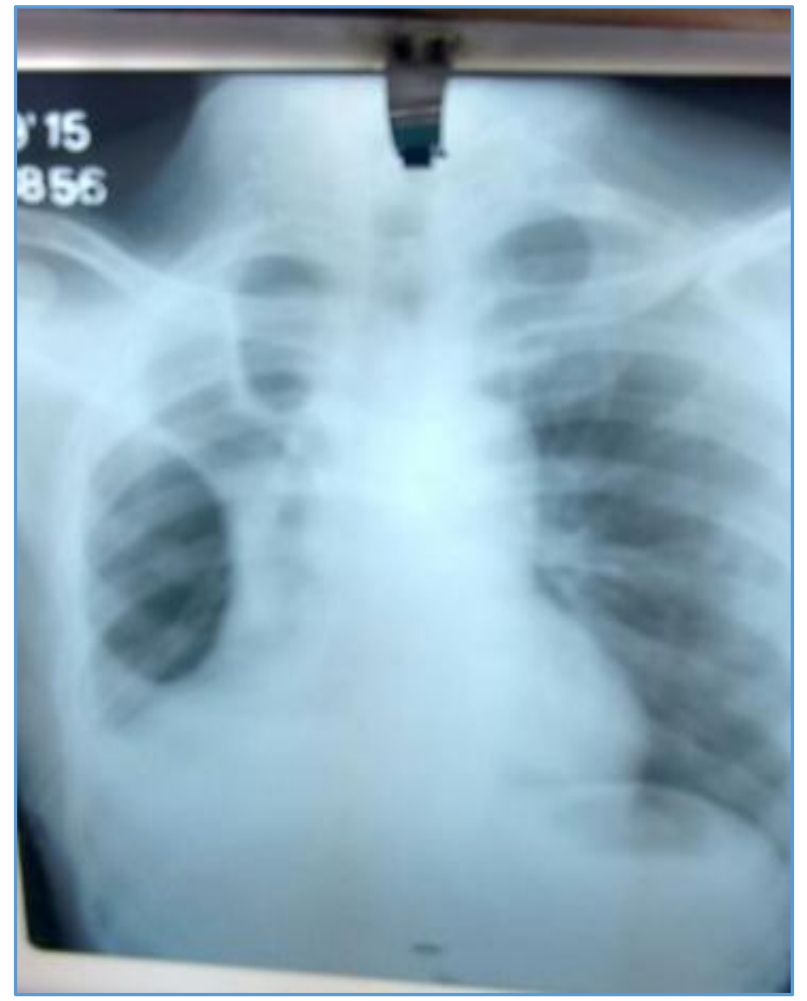

Persistent pneumothorax with BPF with ICD tube in situ after Decortication

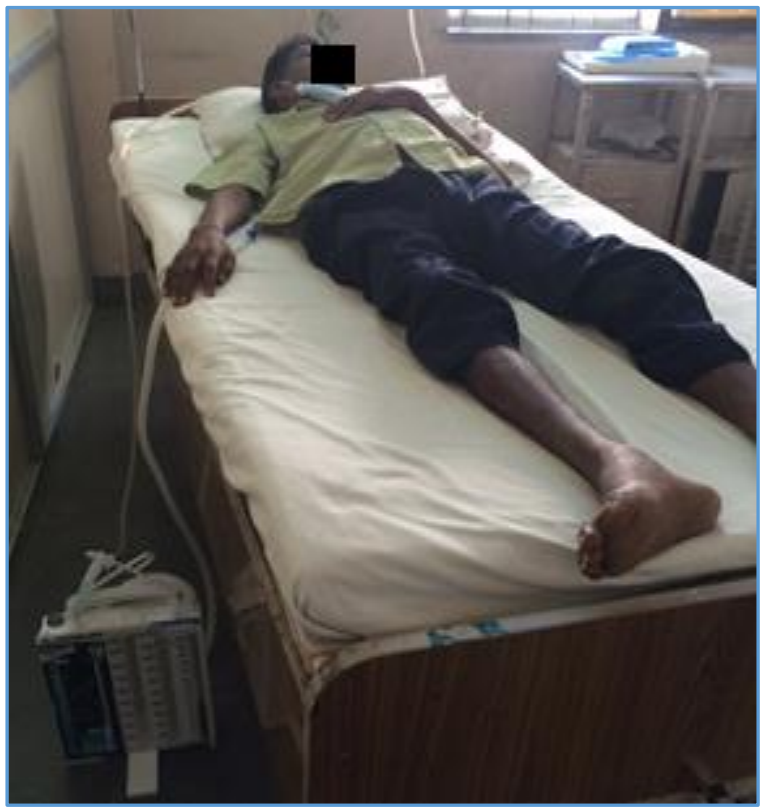

Disposable Water Seal Device (PDU)

\section{REFERENCES}

1. Sahn SA. State of the art. The pleural space diseases. Am Rev Respir Dis 1988;138(1):184-234.

2. Miller KS. "Chest tubes indications, technique, management and complications." Chest Journal 91(2):258

3. Varoli F (1998). Endoscopic treatment of bronchopleural fistulas. Annals of Thoracic Surgery.

4. Judson MA, Sahn SA. The pleural space and surgical procedures. Am J Respir Crit Care Med 1996;153(3):11531165.

5. Xaubet A, Diumenjo MC, Marin A, et al. Characteristics and prognostic value of pleural effusions in tuberculosis. Eur J Respir Dis 1985;66(2):135-140.

6. Randomised clinical trial of chest drainage systems (PDU). Graham ANJ, Cosgrove AP, Gibbons JRP McGuigan JA. Thorax 1992;42:461-2.

7. Laws D, Neville E, Duffy J. Pleural Disease Group, Standards of Care Committee, British Thoracic Society (2003). BTS guidelines for the insertion of a chest drain. Thorax 58 Suppl 2:ii53-9. 